\title{
The Hard X-ray Sky: Recent Observational Progress
}

\author{
Neil Gehrels* and John K. Cannizzo ${ }^{\dagger}$ \\ ${ }^{*}$ NASA/GSFC/ASD/Code 661, Greenbelt, Md 20071 \\ ${ }^{\dagger}$ CRESST/UMBC/NASA/GSFC/ASD/Code 661, Greenbelt, Md 20071
}

\begin{abstract}
The last fifty years have witnessed the birth, development, and maturation to full potential of hard X-ray astrophysics. The primary force driving the history of the field has been the development of space-based instrumentation optimized for getting the maximum science out of observations of high-energy photons from astrophysical sources. Hard X-ray telescopes are leading research in areas such as galactic diffuse emission, galactic transients, and active galactic nuclei.
\end{abstract}

Keywords: <Enter Keywords here>

PACS: 90.

\section{THE EARLY YEARS}

The use of space-based telescopes utilizing scintillator detectors was presaged by proofof-concept balloon flights in the 1960s and 1970s which pioneered the use of these detectors. The first hard X-ray observations were made by balloon experiments with scintillator detectors viewing the sky through collimators. The detectors typically consisted of $\mathrm{NaI}$ scintillation crystals shielded behind by an optically coupled CsI scintillation crystals in a "phoswich" configuration. Each phoswich was enclosed in an annular shield of NaI scintillation crystals providing anticoincidence for soft $\gamma$-ray interactions in the phoswich. A shield would enclose a W slat collimator whose configuration determined the FOV of the phoswich detector.

Among the first space-based experiments possessing gas proportional counters was HEAO-I (1977-1979) [1]. Each counter had a multgrid collimator composed of a stack of etched Mo sheets interleaved with spacer frames. To maintain thermal stability a heat shield made of $2 \mu \mathrm{m}$ Kimfol polycarbonate film and coated with $80 \mathrm{~nm}$ of $\mathrm{Al}$ in front of each collimator. Incident X-rays passed through the heat shield and Mylar film, with the net transmission of the two layers determining the response to soft $X$-rays.

The HEAO $1 \mathrm{~A}-4$ survey [2] detected 44 sources at $40-80 \mathrm{keV}$ above $13 \mathrm{mCrab}$. Fourteen sources were also seen at $80-180 \mathrm{keV}$. Seven of the 44 were extragalactic. The galactic sources included many X-ray pulsars and X-ray transients. A more detailed spectral study of the HEAO $l$ data detected $12 \mathrm{AGN}$ at $E>12 \mathrm{keV}$ and found their spectra to be characterized by a photon index $1.62 \pm 0.04$ [3]. The luminosities of the galaxies ranged from $3 \times 10^{43}$ to $3 \times 10^{45} \mathrm{erg} \mathrm{s}^{-1}$. These workers were also able to place rudimentary constraints on the AGN contribution to the diffuse $X$-ray background. They derived an AGN volume emissivity

$$
B_{\mathrm{AGN}}=1.58 \times 10^{38} \mathrm{erg} \mathrm{s}^{-1} \mathrm{Mpc}^{-3} \mathrm{keV}^{-1} E^{-0.62} .
$$




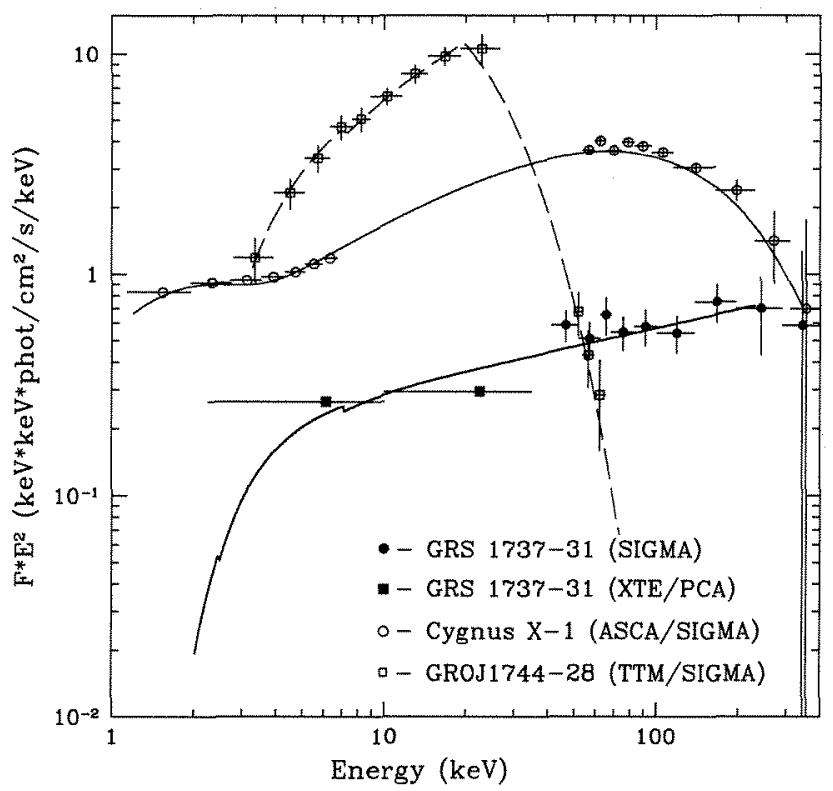

FIGURE 1. Broad-band X-ray spectra [4] of the X-ray nova GRS 1737-31 discovered by GRANAT (filled circles - GRANAT/SIGMA data; filled squares - RXTE/PCA data) compared to two other bright galactic sources, Cyg X-1 (open circles - ASCA and SIGMA) and GRO J1744-28 (open squares - TTM and SIGMA).

\section{GALACTIC ACCRETING SOURCES WITH GRANAT/SIGMA AND BEPPOSAX}

Observations of the galactic center and bulge by GRANAT/SIGMA (1989-1994) showed the spatial distribution of $X$-ray novae to be concentrated toward the galactic center more strongly than the general galactic disk population [4]. It was also shown that if Xray transients follow the distribution of visible mass, their peak luminosity in the band $35-150 \mathrm{keV}$ must lie within a narrow range of $10^{37} \mathrm{erg} \mathrm{s}^{-1}$ [4]. A new source, $\mathrm{X}$ ray nova GRS 1737-31, was found and compared with other bright galactic accreting sources (Figure 1). A GRANAT/SIGMA survey of the galactic bulge region over a four year interval [5] found 14 hard X-ray sources. Their deconvolution of the hard X-rays from the galactic center showed the need for previously unrecognized persistent sources of emission near $\mathrm{Sgr} \mathrm{A}^{*}$. They also found several new transients.

BeppoSAX (1996-2002) observations of Cyg X-1 revealed two spectral states, a soft and a hard state [6]. In both states one clearly saw a soft $X$-ray excess and a broad $\mathrm{Fe} \mathrm{K} \alpha$ line with Compton reflection. The broadband continuum in the soft state was well modeled by a disk blackbody and Compton upscattering of the disk photons by a hybrid, thermal/nonthermal plasma. The primary spectrum in the hard state could be modeled by Compton upscattering of a weak blackbody emission by $\sim 60 \mathrm{keV}$ thermal 


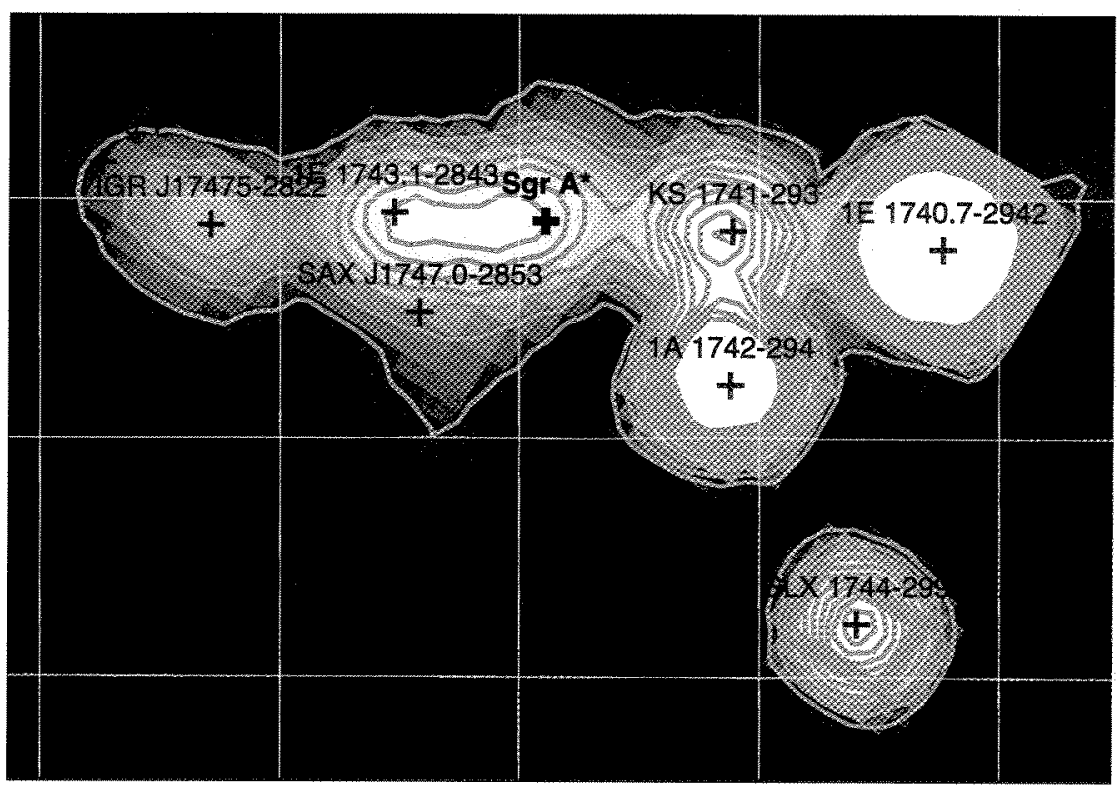

FIGURE 2. INTEGRAL IBIS/ISGRI significance mosaic ( $20-40 \mathrm{keV})$ from 2174 pointings with an effective exposure at the position of Sgr $\mathrm{A}^{*}$ of $4.7 \mathrm{Ms}$ [7].

plasma. The soft excess common to both states could then be explained by thermal Comptonization of the same blackbody emission by another hot plasma cloud with a low Compton parameter. The ratio of the bolometric flux in the soft state to that in the hard state was about 3 .

\section{THE INTEGRAL SKY}

The INTEGRAL (2002-) sky as seen by the IBIS instrument has built upon previous CGRO/COMPTEL results. An all sky map was produced which represented the first complete scan of the entire central median of the galactic plane at energies above 20 $\mathrm{keV}$ with a limiting sensitivity of $\sim 1 \mathrm{mCrab}$. in the central radian [8]. The resultant catalog also included a substantial coverage of extragalactic fields, and encompassed more than 200 high-energy sources. The mean position error for sources detected with significance above $10 \sigma$ was $\sim 40$ arcsec, enough to identify most of them with a known $\mathrm{X}$-ray counterpart [8]. This is important given the high galactic absorption inherent in most earlier surveys. With INTEGRAL, the $20-200 \mathrm{keV}$ galactic emission is resolved into compact sources [9].

Bélanger et al. (2006) studied the persistent high-energy flux from the galactic center using the the IBIS/ISGRI imager on INTEGRAL (Figure 2). For the first time they detected a hard X-ray source, IGR J17456-2901, located within 1 arcmin of Sgr A* 

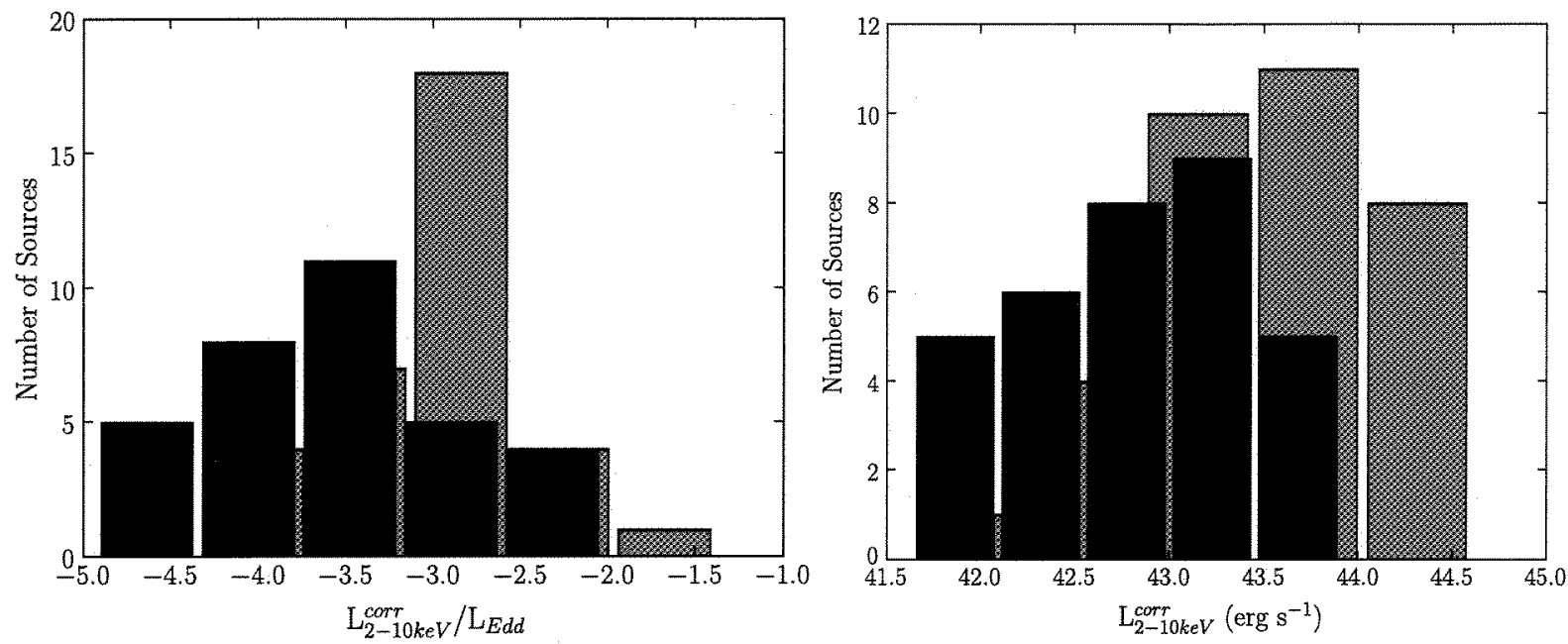

FIGURE 3. Distribution of absorption corrected $2-10 \mathrm{keV}$ luminosity for a Swift/BAT sample of AGN [10] comparing Seyfert 1-1.2 (red) with Seyfert 2 (blue). The luminosities are shown both normalized to their Eddington values, and in $\operatorname{erg~s}^{-1}$. The two distributions are statistically different.

in the energy range $20-100 \mathrm{keV}$, with a $20-400 \mathrm{keV}$ luminosity at $8 \mathrm{kpc}$ of $L=$ $(5.37 \pm 0.21) \times 10^{35} \mathrm{erg} \mathrm{s}^{-1}$. They set a $3 \sigma$ upper limit on the flux at the $e^{-}-e^{+}$ annihilation energy of $511 \mathrm{keV}$ from the direction of Sgr A* at $1.9 \times 10^{-4}$ photons $\mathrm{cm}^{-2} \mathrm{~s}^{-1}$.

\section{EXTRAGALACTIC ASTRONOMY WITH SWIFT/BAT}

A nine month survey using Swift/BAT (2004-) has detected 153 AGN (Figure 3) and provided the first unbiased $\left(N_{H}<10^{24} \mathrm{~cm}^{-2}\right)$ view of local $(z<0.03)$ AGN [10]. "Hidden" AGN - those with scattering fractions $\leq 0.03$ and flux ratios $f_{\text {soft }} / f_{\text {hard }} \leq 0.04$ - constitute a high fraction of the total sample (24\%) [10].

With the accumulated weight of data from wide area surveys carried out in the hard Xray bands by Swift/BAT and other missions, it has recently become possible to calculate detailed and meaningful constraints on the number density and evolution of Comptonthick AGN to explain the X-ray background (Figure 4) [11]. 


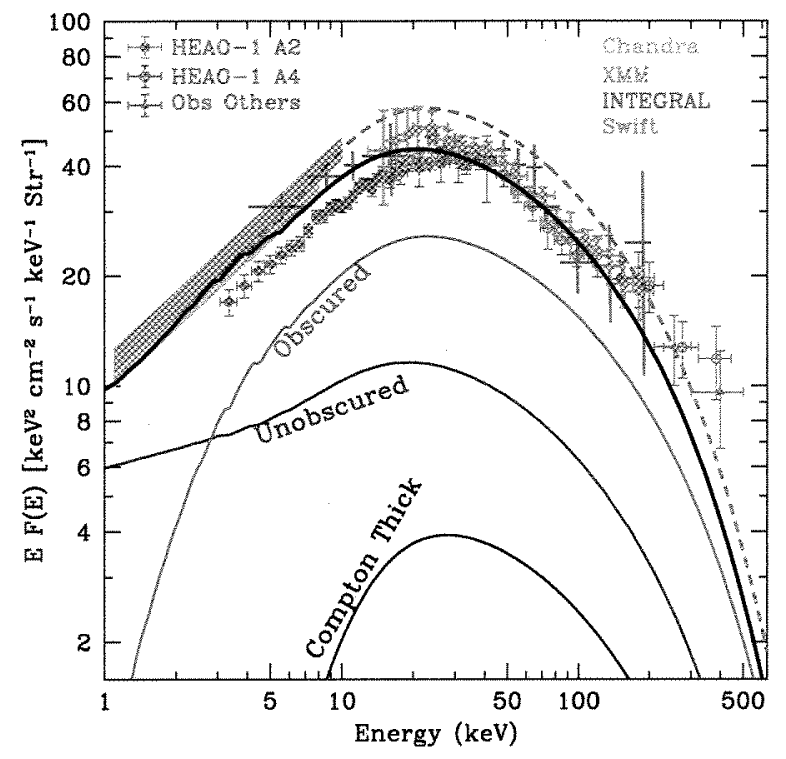

FIGURE 4. Observed spectrum of the extragalactic X-ray background from the indicated telescopes [11]. The dashed gray and thick black solid lines show theoretical XRB spectra from population synthesis models. The red, blue, and thin black solid lines show contributions to the thick black solid line from (i) unobscured AGN, (ii) obscured Compton-thin AGN, and (iii) Compton-thick AGN, respectively.

\section{REFERENCES}

1. K. S. Wood, J. F. Meekins, D. J. Yentis, H. W. Smathers, D. P. McNutt, R. D. Bleach, H. Friedman, E. T. Byram, T. A. Chubb, and M. Meidav, ApJS 56, 507-649 (1984).

2. A. M. Levine, et al., ApJS 54, 581-617 (1984).

3. R. E. Rothschild, W. A. Baity, D. E. Gruber, J. L. Matteson, L. E. Peterson, and R. F. Mushotzky, ApJ 269, 423-437 (1983).

4. S. Trudolyubov, et al., A\&A 342, 496-501 (1999).

5. J. Paul, et al., "SIGMA Survey of the Galactic Bulge Region.," in Seventeeth Texas Symposium on Relativistic Astrophysics and Cosmology, edited by H. Böhringer, G. E. Morfill, and J. E. Trümper, 1995, vol. 759 of New York Academy Sciences Annals, pp. 312-315.

6. F. Frontera, et al., ApJ 546, 1027-1037 (2001).

7. G. Bélanger, A. Goldwurm, M. Renaud, R. Terrier, F. Melia, N. Lund, J. Paul, G. Skinner, and F. Yusef-Zadeh, $A p J$ 636, 275-289 (2006).

8. A. J. Bird, et al., $A p J 636,765-776$ (2006).

9. F. Lebrun, et al., Nature 428, 293-296 (2004).

10. L. M. Winter, R. F. Mushotzky, C. S. Reynolds, and J. Tueller, ApJ 690, 1322-1349 (2009).

11. E. Treister, C. M. Urry, and S. Virani, ArXiv e-prints (2009). 